\title{
Adaptive Decision-Making Scheme for Cognitive Radio Networks
}

\author{
Ismail AlQerm and Basem Shihada \\ CEMSE Division, KAUST, Saudi Arabia, \{ismail.qerm, basem.shihada\}@kaust.edu.sa
}

\begin{abstract}
Radio resource management becomes an important aspect of the current wireless networks because of spectrum scarcity and applications heterogeneity. Cognitive radio is a potential candidate for resource management because of its capability to satisfy the growing wireless demand and improve network efficiency. Decision-making is the main function of the radio resources management process as it determines the radio parameters that control the use of these resources. In this paper, we propose an adaptive decision-making scheme (ADMS) for radio resources management of different types of network applications including: power consuming, emergency, multimedia, and spectrum sharing. ADMS exploits genetic algorithm (GA) as an optimization tool for decision-making. It consists of the several objective functions for the decision-making process such as minimizing power consumption, packet error rate (PER), delay, and interference. On the other hand, maximizing throughput and spectral efficiency. Simulation results and testbed evaluation demonstrate ADMS functionality and efficiency.

Index Terms-Cross-layer Optimization, Transmission Parameters, Decision-making, Genetic Algorithm; Cognitive Radio
\end{abstract}

\section{INTRODUCTION}

The demand for wireless communication is dramatically increasing as more applications are developed. This drives researchers to create new enabling technologies to improve the efficiency of wireless communications to accommodate this demand. Cognitive Radio (CR) is a promising solution to manage radio resources and increase network efficiency. It is considered as an extension of Software Defined Radio (SDR) [1] which is capable to observe, reason, and adapt radio parameters according to application requirements and the radio environment status. CR was first introduced by Mitola in 1999 [2][3] with the vision of building smart radio systems that are able to reconfigure itself and respond to any environment change. Most of the work in the literature [4][5][6] considers Dynamic Spectrum Access (DSA) as the only goal of CR. There are few architectures for radio resource management (RRM), while non of them exploits all TCP/IP layers input for radio adaptation. For any typical cognitive RRM architecture, the QoS requirements dictated by the application layer are essential in the decision-making process. Hence, the application layer impose these requirements such as throughput, delay, PER...etc to the cognitive RRM architecture. The entire network parameters from TCP/IP stack should be considered in order to achieve a more comprehensive decision-making process. Therefore, cross-layer design is necessary to extract the sensory parameters and send them to the decision-making engine to decide about the best solution to configure the radio parameters. The configuration is decided to satisfy the goals of the objective functions. Objective function is a mathematical equation that is evaluated using the radio environment parameters to determine the strength of the available solutions. The concept of cross-layer optimization can be found in [7] and [8].

There are many conventional optimization approaches that are used as the decision-making tool in RRM architectures such as rule based systems [9], fuzzy logic [10], game theory [11], and neural networks [12]. These approaches are capable of providing solutions but they meet severe limitations in parameters adaptation in CR environment. For example, rule based systems are limited to predetermined capabilities according to their own rule set. Fuzzy logic approximated solutions are not based on specific input. Therefore, it does not provide accurate solutions. Neural networks, which is the most popular CR modeling technique includes extensive training to generate observed behavior and it is unstable when constrains are introduced. Game theory that is still at its embryonic stage and is used for interactive decision-making which provides analytical tools to predict the outcome of complex interactions among rational entities based on perceived result. However, the dynamic nature of real networks and impossibility to predict the number of active nodes in real-time makes the implementation of game theory in cognitive radios very complex.

In this paper, we introduce an adaptive decision-making optimization scheme (ADMS) that exploits cross-layer design for optimal parameters selection. It targets various network applications such as power sensitive, emergency, multimedia, and spectrum sharing. ADMS fulfills QoS requirements which include throughput, delay, and PER to increase spectrum efficiency. The following optimization functions form the core of ADMS: minimizing power consumption, PER, interference, delay while maximizing throughput and spectral efficiency. GA [6] [13] is exploited as an optimization methodology. GA fits well with multi-objectives optimization problems for $\mathrm{CR}$ networks as it allows parallelism in the evaluation of the dimensions of objective functions aimed at finding the optimal solution. GA is converges fast, can be implemented over semiconductor devices, can optimize discrete and continuous radio parameters, and works with numerically generated data, experimental data or analytical functions [14].

The novelty of ADMS arises from the following factors:

1) The entire TCP/IP stack layers in the radio environment are considered. This increases the scheme accuracy, 
improves adaptivity, and makes the transmission parameters selection more efficient and convenient.

2) The objectives functions derived in ADMS are comprehensive as they involve all wireless performance metrics such as throughput, delay, PER, spectral efficiency, power, and interference.

3) Thresholds and limits are defined to maximize the fitness scores obtained by the objective functions and to narrow the search space which reduces the complexity of the scheme.

4) ADMS is not limited to point-to-point link optimization. It also includes the environment where cognitive links are non-cooperative, which makes it a good fit to comply with CR networks.

5) The objective functions weights and priorities are adaptive according to the environments conditions and system capability.

6) ADMS facilitates cooperative optimization since it negotiates the selected solution with other nodes in the network. This reduces complexity and avoids conflicts with other nodes selected solutions.

The rest of the paper is organized as follows. In Section II, the decision-making in CR context is introduced. Our derived constrained objectives (fitness) functions for ADMS are also presented. ADMS procedure based on the mentioned objective functions is described in Section III. Section IV comprises performance evaluation, which includes five simulation based evaluation scenarios and hardware implementation. The paper is then closed by the conclusion in Section V.

\section{Constrained Decision-Making in Cognitive RADIO}

Decision-making is a fundamental phase in the cognition cycle state diagram shown in Fig. 1 [2]. It executes optimization and machine learning algorithms to obtain the optimal radio parameters. These parameters are determined by the input from stack layers fetching primitives and QoS requirements received from the application layer. There are several challenges in the

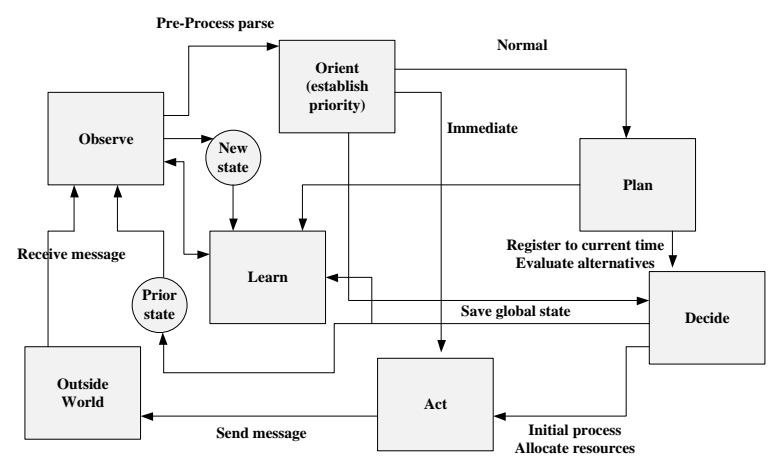

Fig. 1. Cognition Cycle

design of the decision-making scheme that must be considered. First, the optimization goals considered are non-linear over large dimension space. Therefore, the computational complexity for such optimization is a burden on the hardware used for implementation. Second, nonlinear optimization requires a wide internal tuning parameters space. Third, optimization functions that are difficult to create. For example, maximizing throughput may affect other user channel capacity or the overall network performance. Fourth, The tradeoff between the objective function impacts the quality of the decisionmaking. For example, Minimizing PER and Minimizing power consumption are two conflicting objectives. Therefore, the decision-making scheme should be well-developed to tackle the above challenges and improve the decision-making quality. The optimization problem tackled by the decision-making phase is a multi-objective optimization in nature [13][15]. Zitzler [16] provides a brief overview of the multi-objective optimization problems and presents the basic equation for multi-objective decision-making:

$$
\min / \max (Y)=f(x)=\left[f_{1}(x), f_{2}(x) . ., f_{n}(x)\right]
$$

subject to $x=\left(x_{1}, x_{2} . ., x_{m}\right) \in X$ and $y=\left(y_{1}, y_{2} . ., y_{n}\right) \in Y$ in a $n$ dimension space, $f(x)$ is the fitness function for certain

dimension. $X$ is the set of input parameters and $Y$ is the set of dimensions. There are two types of radio parameters defined in the decision-making process: radio environment parameters and transmission parameters. Radio environment parameters represent the input to the decision engine from TCP/IP stack layers. These parameters are exploited to make the decisionmaking engine aware of the surrounding environment. Transmission parameters are the parameters tuned by the cognitive decision engine. Both parameters are determined according to the objective function goal and the TCP/IP layers involved. The transmission parameters configuration values are decided based on the objective function evaluation score (fitness score). We have chosen the following six objectives to be evaluated for resource management: minimizing power consumption, PER, transmission latency, and interference while maximizing throughput and spectral efficiency. Each objective is realized by evaluating its objective function. The objective functions for the above mentioned objectives are normalized to have score between 0 and 1 . Weighted sum approach is employed to find the total objective function based on the six objectives functions. The reason for using weighted sum approach is the tradeoffs between multiple objectives. This approach is convenient because it outputs one scalar value that represents the total fitness score for a solution. In our implementation, we use an adaptive weighted sum where weights of fitness functions are updated periodically and automatically based on QoS requirements and channel conditions changes. The total optimization function based on weighted sum approach is calculated as follows:

$f_{T O}=w_{1} f_{P}+w_{2} f_{T}+w_{3} f_{P E R}+w_{4} f_{D}+w_{5} f_{S E}+w_{6} f_{S I N R}$

where $w_{1}$ to $w_{6}$ are the adaptive weights of the corresponding fitness functions. The fitness functions in (1) are defined as: $f_{P}$ is the power minimization function, $f_{T}$ is the through- 
put maximization function, $f_{P E R}$ is the PER minimization function, $f_{D}$ is the delay minimization function, $f_{S E}$ is the spectral efficiency maximization function, and $f_{S I N R}$ is the interference minimization function. The following sections present the details of the fitness function for each objective.

\section{A. Minimizing Power Consumption}

Power consumption is one of the critical issues that affect mobile terminals sustainability. Battery life information and terminals power consumption are essential factors used for decision-making to reduce power consumption. We define a threshold value for maximum allowed transmission power based on a target PER. Environment and transmission parameters that contribute to power optimization are presented in Table I. These parameters are chosen for the decision-

\begin{tabular}{|l||l||l|}
\hline TCP/IP Layer & $\begin{array}{l}\text { Environment } \\
\text { Parameters }\end{array}$ & $\begin{array}{l}\text { Transmission } \\
\text { Parameters }\end{array}$ \\
\hline MAC Layer & $\begin{array}{l}\text { the ratio of successfully } \\
\text { received packets }\end{array}$ & $\begin{array}{l}\text { Contention Window } \\
\text { Size }\end{array}$ \\
\hline Physical Layer & $\begin{array}{l}\text { Signal to noise ratio } \\
\text { (SNR) }\end{array}$ & Modulation Type \\
& & Modulation Order \\
\cline { 2 - 2 } & & Transmission Power \\
\hline
\end{tabular}

TABLE I

PARAMETERS FOR POWER OPTIMIZATION

making scheme as they strongly affect power consumption. Transmission power is the dominant factor that controls power consumption. Higher modulations orders and channel coding rate increase the processing time and system complexity, thus increasing power consumption. Contention window size impacts the number of retransmissions and this weights on the amount of power consumed. The transmission power consumed per sub-carrier is calculated as follows,

$$
\text { Power Consumption per Sub-carrier }(P i)=P * R s * K
$$

where $P$ is the transmission power, $R s$ is the symbol rate and $K$ is the modulation order. The total power consumed to transmit error-free packets for all sub-carriers is,

$$
P_{s}=S * \sum_{i=1}^{N_{c}} P_{i}
$$

Where $S$ the number of acknowledged packets over the total number of packets transmitted and $N_{c}$ is the number of sub-carriers. The fitness function used to minimize power consumption is calculated as follows,

$$
f_{P}=\left\{\begin{array}{l}
\frac{\left|P_{s}-P^{*}\right|}{P^{*}-P_{r}}, \text { if } P_{r} \leq P_{s} \leq P^{*} \\
1, \text { if } P_{s}<P_{r} \\
0, \text { if } P_{s}>P^{*}
\end{array}\right.
$$

where $P_{r}$ is the minimum power that satisfies QoS requirements and $P^{*}$ is the threshold value of the maximum transmission power per sub-carrier.

\section{B. Maximizing Throughput}

Throughput is another important performance metric in wireless networks. The PER plays the major role in causing throughput degradation of the system. Thus, it should be maintained at an acceptable level. Radio environmental and

\begin{tabular}{|c|c|c|}
\hline TCP/IP Layer & $\begin{array}{l}\text { Environment } \\
\text { Parameters }\end{array}$ & $\begin{array}{l}\text { Transmission } \\
\text { Parameters }\end{array}$ \\
\hline Transport Layer & $\begin{array}{l}\text { ACKs of the received } \\
\text { packets }\end{array}$ & Flow rate \\
\hline \multirow[t]{2}{*}{ Network Layer } & \multirow[t]{2}{*}{ Packet loss ratio } & Number of hops \\
\hline & & Transmission range \\
\hline \multirow[t]{2}{*}{ MAC Layer } & \multirow{2}{*}{$\begin{array}{l}\text { Ratio of successfully } \\
\text { received packets }\end{array}$} & Packet Size \\
\hline & & $\begin{array}{l}\text { Contention window } \\
\text { size }\end{array}$ \\
\hline \multirow[t]{3}{*}{ Physical Layer } & \multirow[t]{3}{*}{ SNR } & Modulation type \\
\hline & & Modulation order \\
\hline & & Symbol rate \\
\hline
\end{tabular}
transmission parameters that are exploited in the decisionmaking process are presented in Table II. The parameters

TABLE II

PARAMETERS FOR THROUGHPUT OPTIMIZATION

selected in Table II are fundamental for throughput optimization. Throughput is proportionally affected by SNR. At high SNR, transmission is often error free, and large packet sizes can be used in order to reduce the MAC layer overhead being transmitted. In addition, the throughput increases as more packets are injected for transmission. The use of fewer hops increases the throughput but weakens the reliability. Throughput $(\mathrm{T})$ can be calculated as follows,

$$
T=\frac{\sum_{i=1}^{N_{c}} \log _{2} M_{i}}{N_{c}}
$$

where $M_{i}$ is the number of bits per symbol. According to (5), it can be deduced that symbol rate and modulation order are proportional to throughput. The following equation calculates the throughput for error-free received packets,

$$
T_{s}=\frac{S T_{k}}{T_{c}} * \frac{\sum_{i=1}^{N_{c}} \log _{2} M_{i}}{N_{c}}
$$

where $T_{k}$ is the time between packet transmission until the ACK is received and $T_{c}$ is the maximum delay after which the packet is considered to be lost. The fitness function for throughput is presented as follows,

$$
f_{T}=\left\{\begin{array}{l}
\frac{\left|T_{s}-T^{*}\right|}{T_{r}-T^{*}}, \text { if } T^{*} \leq T_{s} \leq T_{r} \\
1, \text { if } T_{s}>T_{r} \\
0, \text { if } T_{s}<T^{*}
\end{array}\right.
$$

where $T_{r}$ is the maximum throughput imposed by QoS requirements and $T^{*}$ is the threshold for minimum throughput.

\section{Minimizing Packet Error Rate (PER)}

Error-free transmission is an ultimate goal in wireless communication research. Radio environmental and transmission parameters that are involved in the decision-making process for reliable transmission are presented in Table III. Modulation order affect the probability of errors in the packets received. 


\begin{tabular}{|l||l|l|}
\hline TCP/IP Layer & $\begin{array}{l}\text { Environment } \\
\text { Parameters }\end{array}$ & $\begin{array}{l}\text { Transmission } \\
\text { Parameters }\end{array}$ \\
\hline Network Layer & & Number of hops \\
\hline MAC Layer & $\begin{array}{l}\text { Ratio of successfully } \\
\text { received packets }\end{array}$ & $\begin{array}{l}\text { Contention window } \\
\text { size }\end{array}$ \\
\hline Physical Layer & SNR & Modulation type \\
\cline { 2 - 3 } & Received Signal Strength & Modulation order \\
\cline { 3 - 3 } & & Symbol rate \\
& & Frequency \\
\hline
\end{tabular}

TABLE III

PARAMETERS FOR PER OPTIMIZATION

The number of hops is another essential transmission parameter for transmission reliability. Symbol rate and contention window size negatively impact PER as they increase the bit error probability. Error probability differs from one frequency portion to another. PER is calculated as follows,

$$
P E R=1-\left(1-p_{e}\right)^{N}
$$

where $p_{e}$ is the error probability per bit and $N$ is number of sub-carriers. The value of $p_{e}$ is influenced by several factors such as power to noise ratio, type of noise, attenuation, and fading. The following equation presents the fitness function for minimizing $P E R$,

$$
f_{P E R}=\left\{\begin{array}{l}
\frac{\left|P E R_{s}-P E R^{*}\right|}{P E R^{*}-P E R_{t}}, \text { if } P E R_{t} \leq P E R_{s} \leq P E R^{*} \\
1, \text { if } P E R_{s}<P E R_{t} \\
0, \text { if } P E R_{s}>P E R^{*}
\end{array}\right.
$$

where $P E R_{s}$ the $P E R$ calculated for a given solution, $P E R_{t}$ is the target $P E R$ and $P E R^{*}$ the threshold of maximum tolerated $P E R$.

\section{Minimizing Delay}

Transmission latency is considered as a serious problem in wireless communications especially in real time applications. Table IV, shows the transmission and radio environmental parameters used in delay optimization. Transmission delay

\begin{tabular}{|l||l||l|}
\hline TCP/IP Layer & $\begin{array}{l}\text { Environment } \\
\text { Parameters }\end{array}$ & $\begin{array}{l}\text { Transmission } \\
\text { Parameters }\end{array}$ \\
\hline Transport Layer & Losses of UDP & \\
\hline \multirow{2}{*}{ MAC Layer } & $\begin{array}{l}\text { Packet Retransmission } \\
\text { frequency rate }\end{array}$ & Packet size \\
\cline { 2 - 3 } & $\begin{array}{l}\text { Ratio of successfully } \\
\text { received packets }\end{array}$ & \\
\hline Physical Layer & \multicolumn{2}{|c|}{ TABLE IV } \\
\cline { 2 - 2 } & Modulation type \\
\hline \multicolumn{2}{|c|}{ PARAMETERS FOR DELAY OPTIMIZATION } \\
\hline
\end{tabular}

proportionally increases with file size. Therefore, packet size is an essential parameter for delay optimization. Moreover, modulation order is also considered between the transmission parameters as it has a positive impact on transmission rate which in turn reduces transmission delay. The fitness function to optimize transmission latency is calculated as follows,

$$
f_{D}=L *\left(\frac{\log _{2} M_{\max }}{L_{\min } * \sum_{i=1}^{N_{c}} \log _{2} M_{i}}\right)
$$

where $L$ is packet size and $M_{i}$ is number of bits per symbol.

\section{E. Maximizing Spectral Efficiency}

Maximizing spectral efficiency consists of maximizing the amount of data transmitted over a given bandwidth and is achieved by reducing spectrum occupancy (i.e., the system would transmit high amount of information across narrow bandwidth). Radio environmental and transmission parameters involved in spectral efficiency optimization are presented in Table V. The only practical method to increase the spectral

\begin{tabular}{|l||l||l|}
\hline TCP/IP Layer & $\begin{array}{l}\text { Environmental } \\
\text { Parameters }\end{array}$ & $\begin{array}{l}\text { Transmission } \\
\text { Parameters }\end{array}$ \\
\hline MAC Layer & Bit rate & Bandwidth \\
\hline Physical Layer & \multirow{2}{*}{ SNR } & Frequency \\
\cline { 3 - 3 } & & Modulation order \\
& & Symbol Rate \\
\hline
\end{tabular}

TABLE V

PARAMETERS FOR SPECTRAL EFFICIENCY OPTIMIZATION

efficiency is to increase modulation order, which increases the throughput while keeping the bandwidth fixed. The suitable selection of frequency for data transmission also improves spectral efficiency. Spectral efficiency is calculated as follows,

$$
S_{e}=\frac{R_{b}}{B W}
$$

where $R_{b}$ is the bit rate and $B W$ is the bandwidth. The fitness function to maximize spectral efficiency is presented as follows,

$$
f_{S E}=\left\{\begin{array}{l}
\frac{\left|S_{e}-S E R^{*}\right|}{S E R-S E R^{*}}, \text { if } S E R^{*} \leq S_{e} \leq S E R \\
1, \text { if } S_{e}>S E R \\
0, \text { if } S_{e}<S E R^{*}
\end{array}\right.
$$

where $S E R$ is the maximum spectral efficiency specified by QoS and $S E R^{*}$ is the threshold for minimum spectral efficiency.

\section{F. Minimizing Interference}

Minimizing interference is fundamental in shared spectrum environment like cognitive networks. This objective has the highest priority when spectrum allocation for secondary users in the licensed band is considered. Interference is caused by overlapping transmissions with other users. Therefore, integration over the spectral bandwidth and the total power of overlapping transmissions are necessary to calculate interference. Table VI presents the environment and transmission parameters exploited to minimize interference. As transmis-

\begin{tabular}{|l||l||l|}
\hline TCP/IP Layer & $\begin{array}{l}\text { Environment } \\
\text { Parameters }\end{array}$ & $\begin{array}{l}\text { Transmission } \\
\text { Parameters }\end{array}$ \\
\hline Physical Layer & $\begin{array}{l}\text { Transmission power of } \\
\text { interfering radios }\end{array}$ & Transmission power \\
\hline
\end{tabular}

TABLE VI

PARAMETERS FOR INTERFERENCE OPTIMIZATION

sion power is proportional to interference and interference occurs because of the use of the same frequency portion for transmission. Signal to Interference and Noise Ratio (SINR) is used as the metric to quantify interference and it is calculated as follows,

$$
S I N R=\frac{P}{N+I}
$$


where $P$ is the transmission power, $\mathrm{N}$ is noise $(N=K T B W)$, $I$ is the interference, $K$ is constant, $T$ is the temperature and $B W$ is bandwidth. Interference and SINR are inversely proportional. Therefore, the following equation is used to find the fitness function to minimize interference and maximize SNIR:

$$
f_{S I N R}=\left\{\begin{array}{l}
\frac{\left|S I N R-S I N R_{\max }\right|}{S I N R_{\max }-S I N R_{\min }} \\
\text { if } S I N R_{\min } \leq S I N R \leq S I N R_{\max } \\
1, \text { if } S I N R>S I N R_{\max } \text { no interference } \\
0, \text { if } S I N R<S I N R_{\min } \text { signal is corrupted }
\end{array}\right.
$$

where $S I N R_{\max }$ and $S I N R_{\min }$ are the upper and lower limits of SNIR for the signal to propagate. In the evaluation phase, we have considered different scenarios with different possible weights to demonstrate adaptability.

\section{ADMS: AdAPTIVE DECISION-MAKING SCHEME FOR CR NETWORKS}

The optimization function specified in (1) is not linear since transmission parameters cannot be treated independently. Therefore, tradeoff in the optimization goals must be considered. ADMS aims to this tradeoff by adjusting the weights in (1) dynamically according to the encountered network scenario. Moreover, the objective function may not be used if its objective is out of QoS scope. For example, if real time application is considered, then the delay objective gains the highest weight in (1). All objective functions are linked from a database such that they can be dynamically added and weighted into the fitness evaluation for a specific link condition and performance objectives. ADMS is an evolutionary optimization scheme for decision-making inspired by GA. It starts with a random population of solutions and evolves to reach the optimal solution. Each solution is represented by a trial consisting of a string of bits which corresponds to transmission parameters. The parameters are encoded into bit strings trials using binary encoding and quantized in a priori fashion in a power of two ranges. The trials are evaluated using objective functions to determine the set of trials that fulfill QoS requirements.

The first step in ADMS procedure is to generate an initial random trial used to transmit the first packet. After the packet is successfully received, the trial is evaluated using (1) and the transmission parameters are adjusted. Hence, a new trial is generated to transmit the next packet. The evaluation process is reiterated to generate better solutions until the maximum number of solutions is reached (30 in our implementation). Then, a set of solutions is selected to survive for the next iteration (new population). Reminder Stochastic Sampling is exploited as a selection method as in [17]. During selection, the population of solutions is assumed to form a pie graph where each solution is assigned a space equivalent to its fitness value. Next, an outer roulette wheel is placed around the pie with 30 equally spaced pointers. A single spin of the roulette wheel will now simultaneously pick all 30 members of a median population. After the selection is complete, the median population is created. An Elitism of $10 \%$ is considered to duplicate the best fit set of solutions to the next population to make the algorithm converge faster. Elitism is defined as the process of selecting the best trials from the given solutions set according to the total fitness measure and adding them to the new solutions set without performing crossover and mutation. However, Elitism percentage is maintained low to reduce the the drawbacks of a time consuming search for the optimal solution. After that, crossover and mutation operations are applied on the remaining trials as in [18]. Crossover is the process by which the characteristics of two trials can be reciprocally exchanged to form two new trials [19]. The crossover rates is set to $90 \%$. During Mutation the binary values of a trial are changed from 0 to 1 and vise versa after crossover [20]. The selected mutation rate is kept low at 5\% to avoid damaging the structure of the trial. Finally, a new population is created. Fig. 2 presents the optimization process executed by ADMS. The new population is reevaluated during

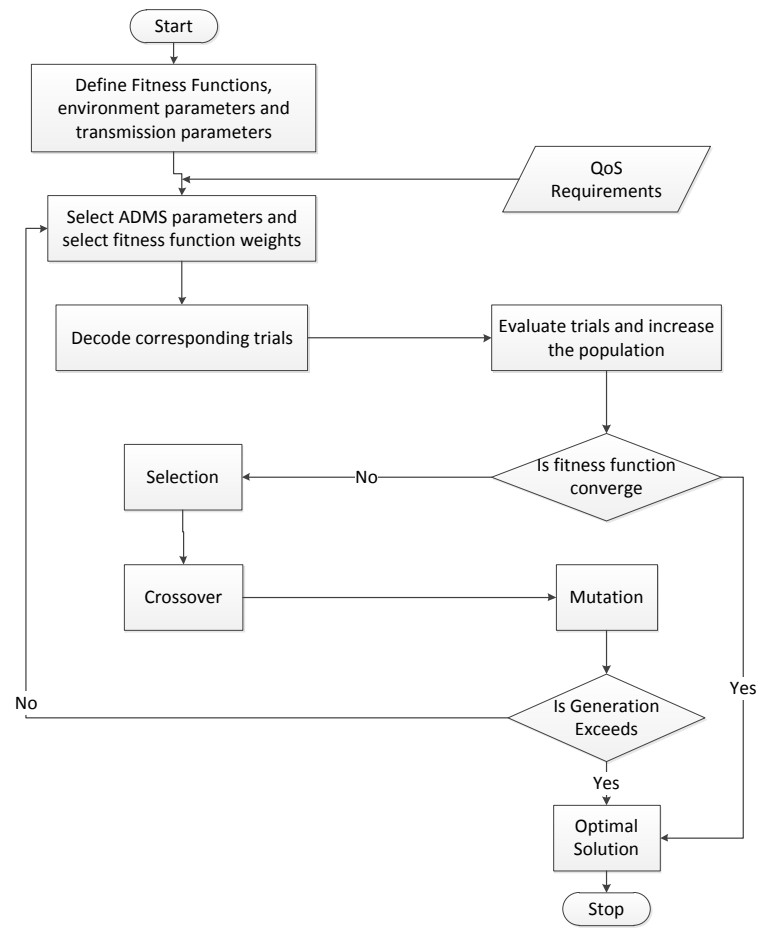

Fig. 2. ADMS Procedure

the transmission of the next 30 packets and the process will continue until the maximum number of iterations is reached (400 in our implementation) or an optimal solution is obtained.

ADMS implementation also ensures that the final solution is compatible with policy regulations. Limits are set in the fitness functions for the transmission parameters such as power and frequency which makes policy verification by the policy layer easier. In addition, our implementation negotiates and exchanges the optimized trials with other nodes in the network. In this way, efficiency is improved and complexity is reduced as other nodes can use this trial to tune their radio in case they encounter similar environment conditions without the need to run ADMS again. Moreover, interference and competition on 
the same resources can be avoided.

\section{EVALUATION}

In this section, the performance of ADMS is evaluated using Matlab and a testbed implementation. We simulated a radio environment that has 60 sub-carriers with constant impulse response channels and half duplex transmission for simplicity. The simulation uses cross-layer transmission parameters configuration with parameters ranges stated in Table VII. The optimization objectives are demonstrated in the following

\begin{tabular}{|c|c|c|}
\hline TCP/IP Layer & Parameter & Range \\
\hline Transport Layer & Flow Rate & Depends on the buffer size \\
\hline \multirow[t]{2}{*}{ Network Layer } & Number of hops & Multi-hop \\
\hline & Transmission range & $\begin{array}{l}\mathrm{d}, \mathrm{d} / 2, \mathrm{~d} / 4 \text { and } \mathrm{d} / 8 \text { where } \\
\mathrm{d} \text { is the distance between } \\
\text { communicating nodes }\end{array}$ \\
\hline \multirow[t]{3}{*}{ MAC Layer } & $\begin{array}{ll}\text { Contention } & \text { window } \\
\text { size } & \end{array}$ & $\begin{array}{l}\text { CWmin } 8 \text { values between } \\
4 \text { and } 64 . \text { CWmax } 8 \text { val- } \\
\text { ues between } 32 \text { and } 4096\end{array}$ \\
\hline & Packet size & 24 bytes to 3072 bytes \\
\hline & Bandwidth & 10 to $50 \mathrm{MHz}$ \\
\hline \multirow[t]{5}{*}{ Physical Layer } & $\begin{array}{l}\text { Modulation type and } \\
\text { order }\end{array}$ & M-PSK and M-QAM \\
\hline & Transmission power & $\begin{array}{l}0.09 \mathrm{mw} \text { to } 5 \mathrm{mw}(-24 \\
\mathrm{dBm} \text { to } 16 \mathrm{dBm})\end{array}$ \\
\hline & Symbol Rate & 1 to $20 \mathrm{Mbps}$ \\
\hline & Frequency & 2400 to $2500 \mathrm{MHz}$ \\
\hline & Channel coding rate & $1 / 2,1 / 3,2 / 3,3 / 4$ \\
\hline
\end{tabular}

TABLE VII

RANGes of Configuration PARAMETERS USED IN Simulation scenarios.

\section{A. General Scenario}

The general scenario incorporates a file transfer between two terminals. Each terminal is equipped with ADMS as a decision-making scheme. The contribution and novelty of ADMS over other schemes are fully demonstrated in this scenario since it involves ADMS ability to adapt transmission parameters to optimal values regardless of the objective functions tradeoffs. All of the TCP/IP layers and objectives functions are involved in this optimization scenario. In this evaluation, we compare ADMS performance against the optimal benchmark in [21]. In addition, we compare it with the GA proposed in [22] for cognitive engine which adopts a similar approach. However, ADMS includes objective functions thresholds used to ensure the optimal transmission parameters selection through the least computation. The weights in [22] for (1) are predetermined on the basis of the overall optimization goal. In ADMS, however, the weights are adaptive to the channel conditions variations and QoS requirements. In [22], only physical and MAC layers are considered in cross-layer optimization and this impacts the solution fitness as the upper layers are not involved. On the other hand, ADMS exploits all stack layers input in the decision-making and extends optimization goals such as delay minimization to make more consistent decisions. The last comparison is with IEEE802.11 standard which does not use dedicated optimization algorithms. Fig. 3 shows the performance of the four schemes as a function of SNR with target $P E R^{*}=10^{-6}$.

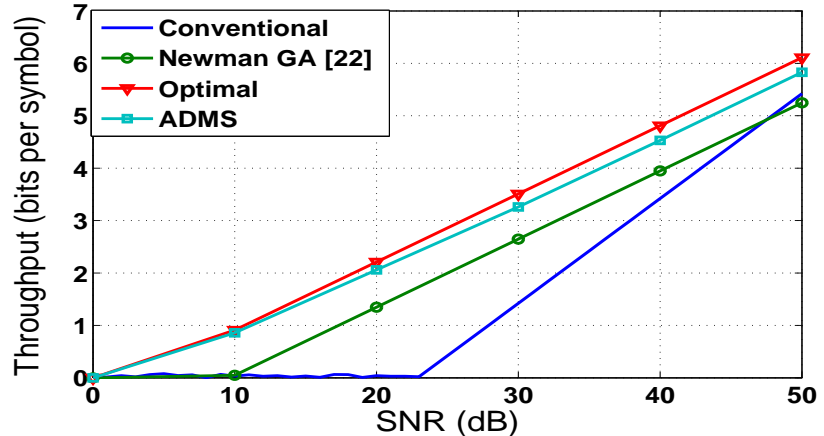

Fig. 3. Throughput of ADMS compared to optimal, Newman [22] and conventional schemes

As shown in Fig. 3, ADMS performance is close to the optimal algorithm when the number of generations is large enough. The difference between ADMS and the optimal algorithm is around $2 \mathrm{dBs}$. Moreover, ADMS outperforms the GA implemented in [22] which is considered as one of the best schemes involving GA as optimization tools for decision-making engine in CR. The conventional approach is penalized by the fact of using the same modulation order over all sub-carriers. In this case, transmission is corrupted by the transmission error over the sub-carriers with the deepest fading. Hence, the performance of this scheme is the worst.

\section{B. Power Optimization Scenario}

In this scenario, power is considered to be the main optimization objective with higher priority as the communicating devices have limited battery capacity. There are two reasons to consider this scenario. First, battery life is one of the main concerns for mobile terminals developers. Second, interference is mainly caused by transmitting at high power level. The scenario includes data transmission between two terminal with the least possible power to extend battery life. Therefore, the weight of power minimization function is initialized to be 0.6 and it then adapts according to network conditions. Fig. 4 (a) presents the fitness score achieved for power consumption optimization. ADMS converges after 60 iterations with random environment conditions at each iteration. The fitness score obtained is relatively high. At the beginning of transmission, full power is used, then using environment awareness, the scheme detects high power consumption as battery life decreases dramatically. Thus, the power consumption is lowered until the power is switched to minimum level to comply with power consumption constraints and objectives. The results obtained in this scenario proves the strength of ADMS as it periodically monitors battery life and reacts accordingly to improve power consumption. In addition, the adaptive weighing mechanism in (1) and the awareness of radio environment makes ADMS not to neglect other optimization objectives even if power has the highest priority.

\section{Emergency Scenario}

This scenario exhibits network environment where all mobile devices want to communicate to report certain incident. Reliability and capacity insurance to accommodate all these 


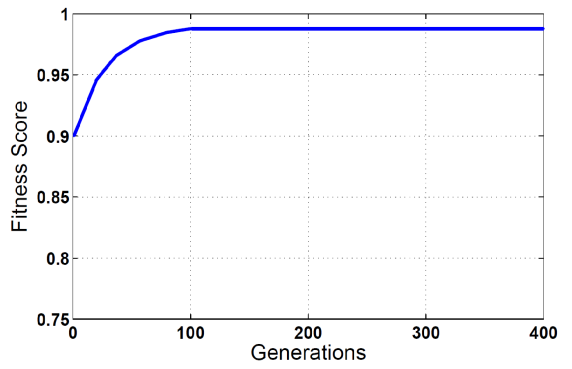

(a)

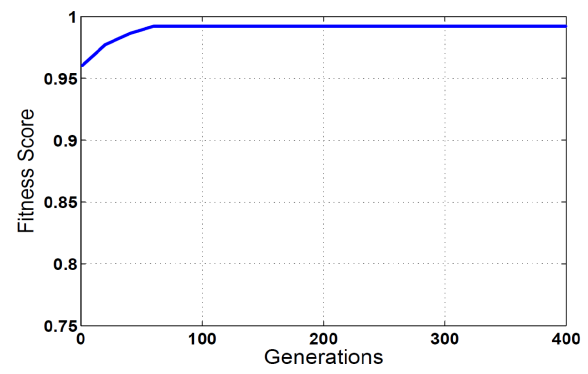

(b)

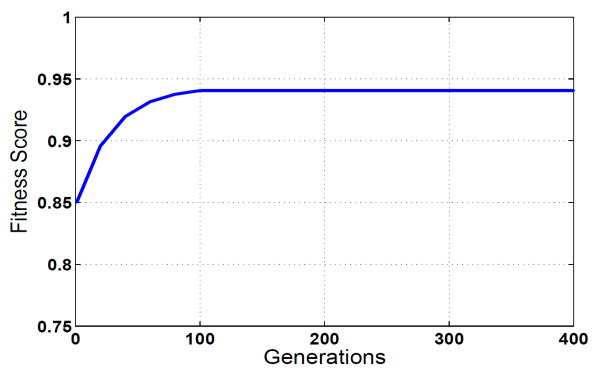

(c)

Fig. 4. Fitness Score for Power Optimization, Emergency, and Multimedia

channels requests are essential optimization objective in the emergency scenario. Therefore, PER minimization is the dominant optimization factor considered in this scenario. Similar scenario is investigated in [22]. However, PER minimization function in (9) used by ADMS is more reliable as it uses thresholds after which communication is considered to be disrupted. This maximizes the tolerated PER and makes ADMS fits more with network conditions variations. The fitness score achieved in the emergency scenario is shown in Fig. 4 (b).

\section{Multimedia Scenario}

The multimedia scenario comprises an online video streaming from a multimedia server, where throughput is the main optimization objective. ADMS throughput fitness function in (7) involves all the stack layers in the optimization process as throughput metric is affected by all of these layer parameters such as modulation index, transmission range, packet size....etc. The achieved fitness score for throughput reached a considerable growth after 70 generations. Fig. 4 (c) shows the throughput fitness score as function of number of generations. Delay is also investigated in this scenario as it is crucial in multimedia applications. Fig. 5 presents the delay encountered against the number of active users with $P E R^{*}=10^{-6}$ and throughput threshold of $1 \mathrm{Mbps}$. It also compares ADMS with "PHY then MAC" optimization approach and the conventional approach (IEEE 802.11). "PHY then MAC" adapts physical layer and MAC layer parameters separately. The parameters of the physical layer are optimized with the algorithm proposed in [21] and the parameters at the MAC layer are optimized through an exhaustive search of all possibilities. ADMS outperforms both approaches as it uses small packet sizes to keep the target throughput and minimize delay.

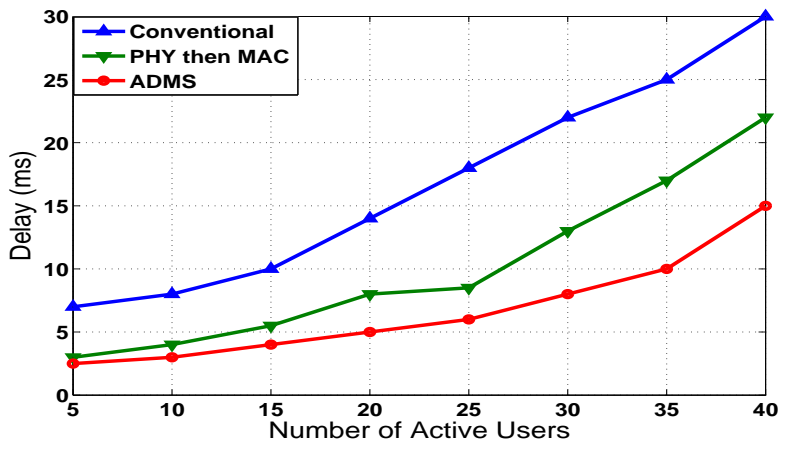

Fig. 5. Encountered Delay in Multimedia Scenario with Active Users

\section{E. Dynamic Spectrum Access Scenario}

Dynamic Spectrum Access (DSA) aims to improve spectrum utilization and limits interference between primary and secondary users. Therefore, spectral efficiency maximization and interference minimization are the main objectives considered. In this scenario, a secondary user tries to access the licensed portion of the spectrum without interfering with primary users. In order to analyze the behavior of ADMS when the spectrum is partially occupied, the average fitness value $f_{P E R}, f_{S E}$ and $f_{S I N R}$ are evaluated over all trials. The goal of ADMS is that all trials in the population correspond to some free channels for two reasons. First, all packets that are transmitted over busy channels are lost and require retransmission, which might significantly reduce the throughput. Second, transmitting over busy channels interferes with other signals which may come from primary users. Therefore, the solutions that correspond to busy channels must be removed at each iteration. The fact that ADMS is aware of the radio environment and uses adaptive weighing factors makes it achieve a remarkable fitness score for both interference and spectral efficiency objective functions. Fig. 6 shows the fitness score of the interference and spectral efficiency fitness functions.

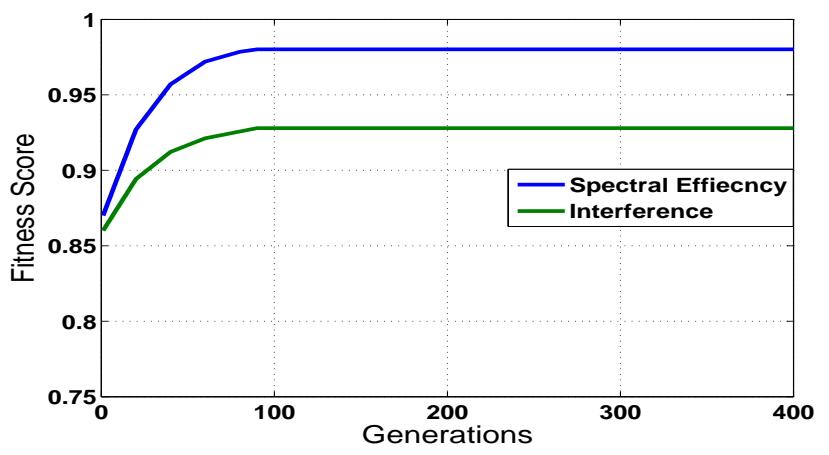

Fig. 6. Fitness Score for Spectral Efficiency and Interference Optimizations in DSA Scenario

\section{F. Hardware Implementation}

Testbed implementation was carried out to demonstrate the strength of ADMS in real life scenario. We used USRP-N210 with RFX-2400 daughter-board as software defined radio (SDR) platform. It operates on wide frequency range between 2.3 and $2.9 \mathrm{GHz}$ and can reconfigure radio with all modulation schemes. The evaluation procedure aims to optimize three 
objectives: minimizing PER, maximizing throughput, and minimizing power consumption. This scenario comprises one user downloading a file from the base station provided that only one radio channel is available and data transmission starts at low data rate and maximum transmission power. The initial random transmission parameters configuration by ADMS were $21 \mathrm{dBm}$ for power, QPSK as modulation type, coding rate equal to $1 / 2$ and data rate of $12 \mathrm{Mbps}$. The initial weights of the objective function in (1) are distributed as follows: 0.4 for throughput maximization, 0.3 for power consumption minimization and 0.3 for PER minimization. When ADMS converges to the optimal set of parameters, the modulation scheme was set to 64-QAM with coding rate of 3/4 to maximize the throughput as it has high priority at the start up stage. The transmission power was set to $4 \mathrm{dBm}$ to fulfill power optimization objective while maintaining PER. The measured throughput was 72 Mbps; 12 Mbps for uplink and 60 Mbps for downlink. Fig. 7 shows power and throughput recorded as function of number of generations when ADMS converged while he measured PER was $10^{-5}$. The conclusion of this scenario is that ADMS managed to improve throughput significantly and minimize power consumption while keeping PER low.

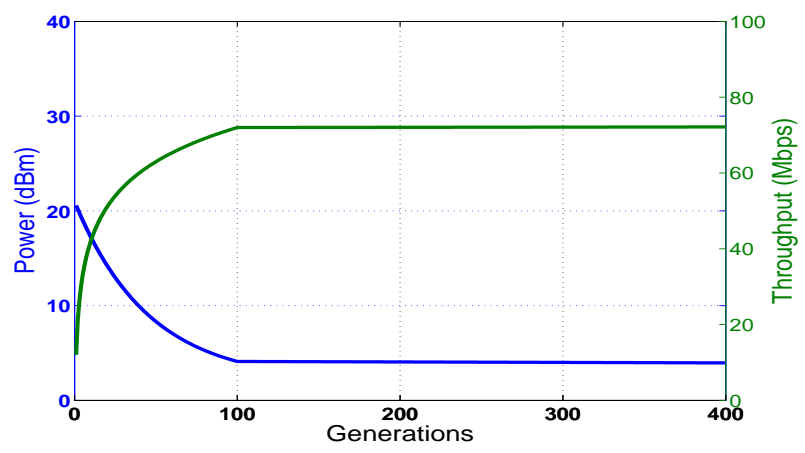

Fig. 7. Throughput and Power Optimization in Testbed

\section{CONCLUSION}

In this paper, we proposed an adaptive decision-making scheme for CR network with multiple sub-carriers. The optimization required the transmitter to be aware of the radio environment. ADMS is employed for decision-making layer to improve the performance of different applications and scenarios such as power sensitive, multimedia, emergency and spectrum sharing. We showed how the decision-making layer can exploit the collected network state information to improve the network performance in terms of all QoS parameters. Our approach is validated by Matlab simulation and testbed implementation using USRP-N210 devices. The results demonstrate the feasibility of ADMS as decision making scheme as they are comparable to the optimal decision making schemes. Future work requires more extensive studies with large-scale networks and considering issues of overall network capacity constraints. One more important issue to consider is complexity of the proposed scheme which includes the scheme complexity, the number of transmission parameters involved, and the time consumed for population generation.

\section{REFERENCES}

[1] A. Luiz Garcia Reis, A.F. Barros, K. Gusso Lenzi, L.G. Pedroso Meloni, and S.E. Barbin, "Introduction to the software-defined radio approach", Latin America Transactions, IEEE (Revista IEEE America Latina), vol. 10, no. 1, pp. $1156-1161$, jan. 2012.

[2] J. Mitola III, Cognitive Radio: An Integrated Agent Architecture for Software Radio, PhD thesis, Royal Institute of Technology (KTH), 2000.

[3] III Mitola, J. and Jr. Maguire, G.Q., "Cognitive radio: making software radios more personal", Personal Communications, IEEE, vol. 6, no. 4 pp. $13-18$, aug 1999.

[4] K.E. Nolan and L.E. Doyle, "Teamwork and collaboration in cognitive wireless networks", Wireless Communications, IEEE, vol. 14, no. 4, pp. $22-27$, august 2007.

[5] Petri Mähönen, Marina Petrova, Janne Riihijärvi, and Matthias Wellens, "Cognitive Wireless Networks: Your Network Just Became a Teenager", in Proceedings of IEEE INFOCOM 2006, 2006.

[6] C. Rieser, Biologically inspired cognitive radio engine model utilizing distributed genetic algorithms for secure and robust wireless communications and networking, $\mathrm{PhD}$ thesis, Virginia Tech, 2004.

[7] N. Baldo and M. Zorzi, "Cognitive network access using fuzzy decision making", Wireless Communications, IEEE Transactions on, vol. 8, no. 7, pp. $3523-3535$, july 2009 .

[8] B. Johansson, P. Soldati, and M. Johansson, "Mathematical decomposition techniques for distributed cross-layer optimization of data networks", Selected Areas in Communications, IEEE Journal on, vol. 24, no. 8, pp. $1535-1547$, aug. 2006.

[9] H. Ishibuchi, "Evolutionary multiobjective design of fuzzy rule-based systems", in Foundations of Computational Intelligence, 2007. FOCI 2007. IEEE Symposium on, april 2007, pp. $9-16$

[10] L.A. Zadeh, "Fuzzy logic = computing with words", Fuzzy Systems, IEEE Transactions on, vol. 4, no. 2, pp. 103 -111, may 1996.

[11] K. Akkarajitsakul, E. Hossain, D. Niyato, and Dong In Kim, "Game theoretic approaches for multiple access in wireless networks: A survey", IEEE Communications Surveys Tutorials, vol. 13, no. 3, pp. 372-395, 2011.

[12] Wei-Feng Shi and Shi-Long Xue, "A novel chaotic neural networks and application", in Machine Learning and Cybernetics, 2005. Proceedings of 2005 International Conference on, aug. 2005, vol. 8, pp. $4651-4656$ Vol. 8.

[13] C.M. Fonseca and P.J. Fleming, "Multiobjective optimization and multiple constraint handling with evolutionary algorithms. ii. application example", Systems, Man and Cybernetics, Part A: Systems and Humans, IEEE Transactions on, vol. 28, no. 1, pp. 38 -47, jan 1998.

[14] Sue Ellen Haupt Randy L. Haupt, Practical Genetic Algorithms, Addison-Wesley Longman Publishing Co., Inc., 3nd edition, 2004.

[15] Haiming Lu and G.G. Yen, "Multiobjective optimization design via genetic algorithm", in Control Applications, 2001. (CCA '01). Proceedings of the 2001 IEEE International Conference on, 2001, pp. $1190-1195$.

[16] E. Zitzler and L. Thiele, "Multiobjective evolutionary algorithms: a comparative case study and the strength pareto approach", Evolutionary Computation, IEEE Transactions on, vol. 3, no. 4, pp. 257 -271, nov 1999.

[17] Darrell Whitley, "A genetic algorithm tutorial", Statistics and Computing, vol. 4, pp. 65-85, 1994.

[18] C.M. Fonseca and P.J. Fleming, "Multiobjective optimization and multiple constraint handling with evolutionary algorithms. i. a unified formulation", S IEEE Transactions on systems, Man and Cybernetics, Part A: Systems and Humans, vol. 28, no. 1, pp. 26 -37, jan 1998.

[19] Ouzhan Hasanebi and Fuat Erbatur, "Evaluation of crossover techniques in genetic algorithm based optimum structural design", Computers and Structures, vol. 78, no. 13, pp. 435 - 448, 2000.

[20] Reeves C R and J E Rowe, Genetic Algorithms: Principles and Perspectives A Guide to GA Theory, Kluwer Academic Publishers, 2003.

[21] R.F.H. Fischer and J.B. Huber, "A new loading algorithm for discrete multitone transmission", in Global Telecommunications Conference : The Key to Global Prosperity, nov 1996, vol. 1, pp. 724 -728 vol.1.

[22] Tim R. Newman, Brett A. Barker, Alexander M. Wyglinski, Arvin Agah, Joseph B. Evans, and Gary J. Minden, "Cognitive engine implementation for wireless multicarrier transceivers", Wirel. Commun. Mob. Comput., vol. 7, no. 9, pp. 1129-1142, Nov. 2007. 